\title{
Essais
}

ESSAIS

Revue interdisciplinaire d'Humanités

Hors-série 6 | 2021

Agrobiodiversité et territoires

\section{Stratégies innovantes pour la sauvegarde et la promotion de l'agro-biodiversité : les projets de l'Association Slow Food International}

Innovative strategies for safeguarding and promoting agro-biodiversity: Projects of the slow food international association

Cristiana Peano, Anna Gregis et Chiara Ghisalberti

\section{OpenEdition}

Journals

Édition électronique

URL : https://journals.openedition.org/essais/7204

DOI : 10.4000 /essais.7204

ISSN : 2276-0970

Éditeur

École doctorale Montaigne Humanités

Édition imprimée

Date de publication : 1 mars 2021

Pagination : 15-36

ISBN : 978-2-492780-00-4

ISSN : 2417-4211

Référence électronique

Cristiana Peano, Anna Gregis et Chiara Ghisalberti, «Stratégies innovantes pour la sauvegarde et la promotion de l'agro-biodiversité : les projets de l'Association Slow Food International », Essais [En ligne], Hors-série 6 | 2021, mis en ligne le 16 mars 2021, consulté le 18 janvier 2023. URL : http:// journals.openedition.org/essais/7204; DOI : https://doi.org/10.4000/essais.7204 


\section{Stratégies innovantes pour la sauvegarde et la promotion de l'agro-biodiversité : les projets de 1'Association Slow Food International}

Cristiana Peano, Anna Gregis, Chiara Ghisalberti

\section{La perte progressive de la biodiversité résultant de l'évolution de la société humaine}

Le terme biodiversité, créé en 1986 à Washington par l'entomologiste Edward $\mathrm{O}$. Wilson, a été résumé à l'article 2 de la Convention sur la diversité biologique $(C D B \text {, Convention on biological diversity, 1992) })^{1}$ comme étant « la variabilité des organismes vivants de toute origine, y compris, entre autres, les écosystèmes terrestres, marins et autres écosystèmes aquatiques et les complexes écologiques dont ils font partie ; cela comprend la diversité au sein des espèces et entre elles ainsi que la diversité des écosystèmes".

De cette diversité, une partie est fondamentale pour l'homme en tant que source de nourriture. En effet, la disponibilité, la quantité et la qualité des aliments sont des paramètres essentiels qui ont accompagné l'homme tout au long de son évolution, influençant les besoins, les cultures et les structures sociales. Pour cette raison, la relation entre l'homme et l'écosystème ne doit pas être considérée comme unidirectionnelle, mais co-évolutive. En particulier, le processus de sélection par l'homme des espèces vivantes, des animaux et des plantes, a déterminé le patrimoine de l'agro-biodiversité qui est identifié comme un facteur déterminant.

Selon la Food and Agriculture Organization of the United Nations (FAO, 1999), "l'agro-biodiversité comprend la variété et la variabilité des animaux, des plantes et des micro-organismes qui sont importants pour l'alimentation et l'agriculture et qui sont le résultat d'interactions entre l'environnement, les ressources génétiques, les systèmes de gestion et les pratiques utilisées par les humains $»^{2}$.

https://www.cbd.int/convention/text/.

2 FAO, 1999a, http://www.fao.org/3/a-y5609e.pdf. 
Les données rapportées par la $F A O^{3}$ montrent que ce patrimoine est aujourd'hui en danger d'être compromis. Il existe environ 7000 espèces de plantes qui peuvent être utilisées par l'homme pour son alimentation, mais seules 150 sont cultivées à grande échelle à cette fin. Douze d'entre elles fournissent près des trois quarts de notre alimentation, et quatre d'entre elles (riz, maïs, blé, pomme de terre) assurent plus de la moitié de l'alimentation quotidienne mondiale. De même, en termes de diversité animale, environ 30 des 50000 espèces de mammifères et d'oiseaux sont largement utilisées pour l'agriculture et l'élevage, et 15 d'entre elles représentent plus de $90 \%$ de la production mondiale de bétail. Par conséquent, la réduction de l' "agro-biodiversité " fait partie d'un phénomène plus large de perte de biodiversité mondiale, due à la dégradation des écosystèmes et à la perte d'habitats naturels $\mathrm{s}^{4}$.

Le processus de sélection des ressources comestibles par l'homme, a fortement aggravé le maintien des ressources naturelles, au point qu'il est nécessaire de mener des activités nombreuses et diversifiées pour sauvegarder, préserver et promouvoir la biodiversité, utile à la protection générale de l'écosystème terrestre.

Depuis des décennies, la société humaine, dans son évolution constante en termes démographiques, spatiaux et technologiques, s'est identifiée comme la seule espèce capable de dominer la nature, en la façonnant selon ses besoins. L'application d'un modèle unique, le modèle capitaliste, a provoqué une transformation évidente de la planète Terre, et l'ère dans laquelle nous vivons est identifiée par la communauté scientifique comme l'Anthropocène. La définition originale, diffusée par le biologiste Eugène F. Stoermer et divulgué par le prix Nobel de chimie P. Crutzen, définit l'Anthropocène comme « l'âge géologique dans lequel l'environnement de la Terre, compris comme l'ensemble des caractéristiques physiques, chimiques et biologiques dans lesquelles la vie a lieu et évolue, est fortement conditionné à l'échelle locale et mondiale par les effets de l'action humaine $"^{5}$.

Ce processus est en effet crucial pour la vie de l'être humain, qui en subit les conséquences, directement ou indirectement, mais il est aussi fortement déterminant pour toutes les autres espèces vivantes qui habitent l'écosystème de la Terre. Pour souligner cette situation, il est important de se référer au fait que presque tous les 17 Objectifs de Développement Durable de l'Agenda 2030 des Nations Unies ont pour thème la biodiversité et la protection de

3 The state of the world's biodiversity for food and Agriculture, FAO Commission on genetic resources for food and agriculture assessment, 2019. link http://www.fao.org/news/story/it/ item/1181477/icode/.

4 http://www.fao.org/family-farming/detail/en/c/1245425/.

5 https://www.nature.com/articles/415023a - Geology of mankind, P. Crutzen, in Nature, 415, $23(2002)$. 
l'environnement et que l'objectif 15 (Vie sur Terre) souligne la nécessité de mettre en œuvre des plans nationaux pour la protection, la restauration et l'utilisation durable de l'écosystème terrestre, la gestion durable des forêts, la lutte contre la désertification, l'arrêt de la dégradation des sols et l'arrêt de la perte de la diversité biologique. ${ }^{6}$

C'est donc dans la relation entre la nature et la culture, entre l'homme et le non-humain, que se cache le rôle fondamental de la préservation et de la sauvegarde de la biodiversité, et donc de l'agro-biodiversité.

\section{Des Systèmes Agricoles Diversifiés pour préserver l'agro-biodiversité}

Au cours des cinquante dernières années, de nombreux accords internationaux ont été négociés pour tenter d'assurer la conservation et la promotion de la biodiversité et de l'agro-biodiversité ${ }^{7}$ afin de souligner à quel point elle est fondamentale dans la définition de l'état de santé d'un système. Un écosystème caractérisé par quelques espèces individuelles appartenant aux groupes les plus résilients est plus vulnérable et plus sujet à des risques tels que la désertification, la colonisation par des espèces exotiques, la perturbation des services de base des écosystèmes et l'insécurité alimentaire, avec des conséquences et des répercussions graves, en particulier dans des contextes socioéconomiques fragiles ${ }^{8}$.

La biodiversité - du gène, au niveau élémentaire, à l'écosystème, à un niveau plus complexe - est donc un élément clé pour définir la résistance et la résilience du système. Le premier terme fait référence au degré de résistance à une perturbation qui l'éloigne de son état d'équilibre initial, et le second à la capacité d'un système à revenir à des normes minimales après une perturbation, la capacité à récupérer après une chute.

Une approche particulièrement intéressante en ce sens est celle des Diversified Farming Systems (DFS), introduite par un groupe d'auteurs en 2012 dans la revue Ecology and Society 9 . Ces modèles s'accordent sur l'accent à mettre sur la

6 https://sustainabledevelopment.un.org/sdg15.

7 Quelques exemples sont le protocole de Nagoya : https://www.admin.ch/opc/it/classifiedcompilation/20130833/201502170000/0.451.432.pdf ; le traité international sur les ressources phytogénétiques pour l'alimentation et l'agriculture (ITPGRFA) : http://www.fao. org/plant-treaty/en/; et à la suite d'une réunion sur la biodiversité et les services écosystémiques tenue en 2010 à Butan, la plateforme intergouvernementale sur la biodiversité et les services écosystémiques (IPBES) : https://ipbes.net/.

8 Nadia Tecco, Vincenzo Girgenti, Cristiana Peano, Francesco Sottile, "The role of diversity and diversification for resilient agricultural systems", III, Congress of the Italian University Network for Development Cooperation, 2013.

9 Claire Kremen, Alastair Iles, Christopher Bacon, "Diversified farming systems: an agroecological, systems-based alternative to modern industrial agriculture", in Ecology and Society, 17(4), 2012, p. 44. 
production, les connaissances et les pratiques locales des agriculteurs, c'est-à-dire sur des approches qui limitent les externalités négatives. L'objectif est d'améliorer la durabilité et la résilience des systèmes agricoles, en contribuant à des questions clés telles que la sécurité alimentaire et la santé.

Linnovation du modèle $D F S$ consiste à aller au-delà des concepts souvent malmenés d'agriculture durable, multifonctionnelle et biologique, dans une tentative de créer un dénominateur commun par la réalisation d'objectifs minimums parmi les diverses approches qui cherchent à obtenir une agriculture de qualité tout en préservant les services écosystémiques pour les générations actuelles et futures, à une époque de changement climatique, d'inégalités sociales et de dégradation croissante de l'environnement. Tout cela à la lumière de la diversité des contextes écologiques, socio-économiques, historiques et politiques dans lesquels les systèmes agricoles se sont développés et continuent d'évoluer ${ }^{10}$. Cette approche permet donc de travailler sur un certain nombre d'attributs qui servent nécessairement à définir un système durable. La biodiversité devient l'ingrédient crucial de la résilience d'un agroécosystème, en la plaçant dans une stratégie caractérisée par la résilience comme une approche et non comme une fin en soi. ${ }^{11}$

Il est important de souligner que le rôle de la composante sociale ne doit pas non plus être sous-estimé. Selon la vision de l'écologie humaine, en effet, les écosystèmes sont fortement interconnectés avec le tissu social dans lequel ils se développent, et cela est particulièrement vrai pour l'agriculture en raison de la connexion permanente entre les sociétés humaines et les écosystèmes, résultat d'une co-évolution entre la nature et la culture. ${ }^{12}$

\section{Slow Food et la Fondation pour la Biodiversité : une proposition innovante pour la protection de l'agro-biodiversité}

Sur la scène internationale, il existe de nombreux projets locaux et non locaux visant à la conservation et à la gestion durable des écosystèmes et des habitats présentant un degré élevé de diversité, ainsi qu’à la conservation de la biodiversité et de l'agro-biodiversité. Nombre d'entre elles reposent sur le concept selon lequel la protection et l'amélioration véritables de la diversité

10 Fanny Boeraeve, Nicolas Dendoncker, Marc Dufrêne, Contribution of diversified farming systems to the delivery of ecosystem services. $2^{e}$ Congrès interdisciplinaire du Développement durable (20-22 mai 2015), https://cidd2015.sciencesconf.org/51689/document.

11 Claire Kremen and Albie Miles, Ecosystem Services in Biologically Diversified versus Conventional Farming Systems: Benefits, Externalities, and Trade-Offs, Ecology and Society, vol. 17, $\mathrm{n}^{\circ} 4$, 2012.

12 Christopher M. Bacon, Christy Getz, Sibella Kraus, Maywa Montenegro and Kaelin Holland, The Social Dimensions of Sustainability and Change in Diversified Farming Systems, Ecology and Society, vol. 17, n 4, 2012. 
biologique ne peuvent se faire que par une action intersectorielle, qui s'intéresse à l'écosystème dans toutes ses différentes composantes, tant naturelles qu'humaines. ${ }^{13}$

Un exemple concret que nous voulons analyser ici est celui de l'association internationale Slow Food : une organisation éco-gastronomique à but non lucratif fondée en 1986 en Italie. Grâce au travail de son réseau de producteurs, de coproducteurs et de la société civile et à sa structure démocratique, le mouvement œuvre à la défense des droits primaires, en premier lieu le droit à l'alimentation, à l'environnement et aux biens communs. Au fil des ans, le mouvement en est venu à créer un réseau de communautés locales présentes dans plus de 160 pays à travers le monde, qui, grâce à une collaboration et une coopération constante, mènent diverses activités et initiatives, en accord avec la vision du mouvement. À la base de tous les projets se trouve la préservation de la biodiversité, de l'ethno-diversité et du droit à la souveraineté alimentaire, le respect des identités culturelles, des peuples indigènes et de leurs cultures gastronomiques et pratiques agricoles respectives, en stimulant une réduction judicieuse des déchets. Elle se mobilise également pour la protection du bien-être des animaux, la protection de l'environnement, des paysages, des ressources naturelles et la lutte contre le changement climatique. En favorisant la réduction de la distance entre le producteur et le consommateur, elle encourage les modèles d'économies locales en créant des projets de coopération internationale dans les pays du Sud (Slow Food, 2017), des campagnes de sensibilisation et des activités d'advocacy; elle coordonne et soutient également des initiatives au niveau local en collaboration avec les acteurs sociaux. Enfin, elle organise des activités dans les écoles pour des activités éducatives liées à la nutrition, au goût et à la culture gastronomique.

L'accent initial de l'association sur les plaisirs de la nourriture et du goût est maintenant accompagné de questions environnementales. La prise de conscience croissante des questions alimentaires a donc permis le développement de son slogan le plus connu : «Bon, propre et juste ». ${ }^{14}$

Il n'existe pas de définition univoque d'un système alimentaire durable dans la littérature, ni de critères uniques pour évaluer si un système alimentaire est durable ou non dans son intégralité. En fait, il existe différentes évaluations selon les domaines d'application, par exemple l'évaluation de l'impact des systèmes agricoles durables, la gestion des terres et des paysages, la gestion des ressources naturelles. ${ }^{15}$

13 Nadia Tecco, Vincenzo Girgenti, Cristiana Peano, Francesco Sottile, "The role of diversity and diversification for resilient agricultural systems", III, Congress of the Italian University Network for Development Cooperation, Turin, 2013.

14 Carlo Petrini, Buono, pulito e giusto, Slow Food Editore, 2a ed., 2016.

15 Pacini et al., 2009 ; Van der Werf e Petit, 2002 ; Smith e Dumanski, 1994 ; Vereijken, 1999 ; Van Mansvelt e Van der Lubbe, 1999 ; Weersink et al., 2002 ; Lopez-Ridaura et al., Dei principi dell'agricoltura biologica (IFOAM) e della politica alimentare e dell'etica, 2002 (Lang et al., 2009). 
Plus généralement, cependant, on peut dire qu'un système agroalimentaire durable correspond à une production de qualité qui est économiquement durable, écologiquement viable, socialement juste et culturellement acceptable.

L'ambition des projets de Slow Food, en particulier ceux qui appartiennent à la Fondation Slow Food pour la Biodiversité, est précisément d'aller dans cette direction, en soutenant une production de qualité souvent menacée d'extinction, en protégeant des territoires et des écosystèmes uniques, en récupérant des méthodes de transformation traditionnelles et en sauvegardant les races indigènes et les variétés végétales locales. ${ }^{16}$

L'approche de la conservation est orientée vers des interventions qui respectent non seulement les équilibres éco-systémiques mais aussi les traditions et les cultures en danger d'extinction. La relation entre les connaissances traditionnelles et les ressources naturelles a été étudiée en profondeur pendant de nombreuses années, et les développements liés à la mise en œuvre de la $C D B^{17}$ ont également conduit à des approches sensiblement différentes de la gestion de la biodiversité.

Il en ressort que la conservation de l'agro-biodiversité est un processus étroitement lié à son utilisation et à sa consommation par sa communauté ou plus généralement par les personnes en contact avec elle. Cela a clairement des implications importantes pour toutes les espèces, variétés et écotypes d'intérêt nutritionnel général qui, au fil du temps, ont joué un rôle constant dans l'alimentation quotidienne.

Par conséquent, les personnes chargées de leur entretien et de leur reproduction sont considérées comme les gardiens d'une partie importante de ce patrimoine local, et sont devenues la preuve vivante des méthodes et des connaissances traditionnelles. Il s'agit donc d'un patrimoine qui doit nécessairement être préservé afin de contribuer au maintien d'une culture de consommation locale et durable qui favorise la biodiversité et renforce le tissu social local. ${ }^{18}$

\section{La stratégie intégrée dans l'action de Slow Food}

L'alimentation a toujours été au cœur de la philosophie de Slow Food, mais si au début l'accent était davantage mis sur la gastronomie à travers la préservation des traditions et des saveurs originales, ces dernières années l'association s'est activement consacrée aux questions de protection de l'en-

16 Cristiana Peano, "I progetti della fondazione Slow Food per la biodiversità: un modello per valorizzare cibi locali di qualità", in Geografia da e para a cooperação ao desenvolvimento territorial: experiencias brasileiras e italianas, 2011, p. 293-313, Ed. Outras Expressōes.

17 Plan stratégique pour la biodiversité 2011-2020, https://www.cbd.int/decision/cop/?id=12268.

18 Francesco Sottile, M. Beatrice Del Signore, Serena Milano, Cristiana Peano, Vincenzo Girgenti, "Cultural diversity and conservation of indigenous and native diversity", III, Congress of the Italian University Network for Development Cooperation, 2013. 
vironnement, d'économie locale et de durabilité agroalimentaire. Malgré sa complexité, il est possible d'identifier ${ }^{19}$ une stratégie d'action qui répond à une approche holistique, qui comprend cinq thèmes centraux qui guident Slow Food dans sa planification et son activisme.

1. Protection de la biodiversité et de la multifonctionnalité des agroécosystèmes. Slow Food s'intéresse à la sauvegarde de la biodiversité de la production agricole, de la biodiversité alimentaire et de la biodiversité sauvage. Tout ce que l'homme a soigneusement sélectionné et sauvegardé pour son alimentation en 10000 ans est aujourd'hui constamment perdu, précisément à cause de son comportement. C'est un problème énorme non seulement pour les pays riches, qui voient leur richesse gastronomique, leurs traditions et leurs pratiques agricoles constamment menacées ; mais c'est encore plus grave pour les pays pauvres, qui voient leur souveraineté alimentaire menacée.

2. Multi-dimensionnalité du concept de qualité. Compte tenu de l'extrême complexité du terme " qualité " dans le secteur agroalimentaire, il est nécessaire de l'analyser selon une approche multidimensionnelle. La qualité peut être comprise comme hygiénique-sanitaire, environnementale, au point d'inclure des aspects historico-culturels, et donc la qualité d'un point de vue social et territorial. D'autre part, Slow Food a décidé de soutenir l'idée de "qualité narrée » en incluant de multiples aspects qui ne peuvent être séparés les uns des autres, elle doit donc être communiquée à travers un récit exhaustif qui détermine le statut qualitatif d'une production, d'une variété ou d'une race spécifique. La qualité narrative de Slow Food se forme par la coexistence du bon, du juste et du propre.

Pour le bon, nous nous référons au goût décliné en saveur et en connaissance. Le goût est lié à la saveur, " en tant que sensation individuelle de la langue et du palais : l'expérience est par définition subjective, insaisissable, incommunicable $"^{20}$; la connaissance, " est l'évaluation de ce qui est bon ou mauvais ". Compte tenu des fortes menaces qui pèsent aujourd'hui sur le système alimentaire mondial, si l'on considère le profil nutritionnel dégradé, la suppression de l'agriculture locale par un type d'agriculture industrielle et, plus généralement, la standardisation des produits, il est donc nécessaire de rééduquer la sensorialité et de faire réapparaittre les cultures gastronomiques traditionnelles. C'est pourquoi le concept de bien ne peut être réduit à une seule signification, mais doit inclure une pluralité d'aspects tels que la sensorialité, la nutrition, la diversité, la culture, la sphère émotionnelle, l'artisanat. ${ }^{21}$

19 Egidio Dansero, Cristiana Peano, Carlo Semita, Nadia Tecco, "Il modello delle comunità del cibo nell'azione di Slow Food in Africa. Modalità operative e indicazioni per la valutazione e il monitoraggio delle attività", 2015, https:/www.slowfood.com/sloweurope/wp-content/ uploads/rapporto_11 maggio.pdf.

20 Mario Montanari, Il cibo come cultura, Economica Laterza, Roma, Laterza Editore, 2004.

21 Slow Food Europe, The Meaning of Quality for Slow Food - Internal Working Paper, 2018. 
Le concept de propreté fait référence à la durabilité environnementale de l'ensemble de la chaîne d'approvisionnement, de la production dans les champs à sa consommation sur la table, "from farm to fork ". Ce terme fait référence à la saisonnalité, au lieu, à la santé, au bien-être des animaux et à l'intégrité. Une multiplicité d'aspects qui supposent un équilibre entre les écosystèmes, la protection de la biodiversité et des ressources naturelles, la sauvegarde de la santé humaine et de chaque être vivant.

Enfin, même le concept de droit ne repose pas sur une seule racine mais considère une multiplicité d'aspects : justice économique et sociale, respect des travailleurs et de leur bien-être, salaires adéquats, accès à la nourriture, solidarité, transparence, bien-être des animaux. ${ }^{22}$ Une étape importante vers la reconnaissance des droits des agriculteurs et des travailleurs du secteur rural a été franchie avec la "Déclaration des Nations Unies sur les droits des agriculteurs » en 2017. Bien qu'il vise à défendre leurs droits et donc à réduire les inégalités, Slow Food soutient qu'il reste beaucoup à faire pour rendre justice au monde de l'agroalimentaire. Pour développer un système alimentaire durable, il est nécessaire de réorganiser la chaîne de distribution qui, grâce à sa structure financière, s'est articulée sur de nouveaux acteurs, les intermédiaires, qui ont complètement brisé le lien entre le producteur et le consommateur. Pour qu'il y ait transparence des prix et une rémunération correcte des travailleurs, il est essentiel de raccourcir la chaîne d'approvisionnement et de soutenir ainsi l'agriculture à petite échelle. ${ }^{23}$

3. Redéfinition de la relation producteur-consommateur. Grâce à la narration de la qualité, le consommateur prend activement conscience de l'ensemble du processus de production, générant et reconstruisant une relation transparente et digne de confiance. Dans ce rapprochement entre producteur et consommateur, de nature plus conceptuelle mais aux conséquences pratiques évidentes, Slow Food promeut également une réduction de la distance physique entre les deux catégories de sujets. En favorisant une chaîne d'approvisionnement courte, Slow Food offre la possibilité de développer les économies locales en s'appuyant sur la juste valeur du produit commercialisable et en définissant un prix équitable, tant pour le producteur que pour le consommateur, qui dans la terminologie de Slow Food est défini comme " co-producteur".

4. Développement local et rural. Le développement local - reconnu au sens large comme le résultat d'un processus d'interaction entre des acteurs locaux (publics et privés) qui partagent implicitement ou explicitement certaines

22 Slow Food, "Slow Food's Contribution to the Debate on the Sustainability of the Food System", 2013, https:/www.slowfood.com/sloweurope/wp-content/uploads/ING-food-sust.pdf.

23 Egidio Dansero, Cristiana Peano, Carlo Semita, Nadia Tecco, "Il modello delle comunità del cibo nell'azione di Slow Food in Africa. Modalità operative e indicazioni per la valutazione e il monitoraggio delle attività", 2015, https://www.slowfood.com/sloweurope/wp-content/ uploads/rapporto_11 maggio.pdf. 
visions du développement pour la valorisation des ressources territoriales et des « richesses » de diverses natures (matérielles et immatérielles) dont ils disposent est devenu une partie intégrante de la stratégie de Slow Food. À côté de cela, on trouve les concepts de territorialité et d'approche bottom-up du développement. Le concept de territorialité reflète la vision d'un territoire comme un ensemble de relations entre les communautés établies, leurs cultures et l'environnement. Par conséquent, la préservation des écosystèmes ne s'oppose pas à celle de la société et de son développement local (également économique), mais considère les composantes naturelles et socio-économiques comme deux aspects inextricables d'une dynamique qui doit être considérée dans sa globalité. Dans le concept d'une approche bottom-up du développement, l'action des Slow Food semble s'inspirer en particulier des théories du développement rural endogène et des expériences de développement rural, qui voient dans la valorisation de l'identité culturelle une réponse à la crise du modèle de production industrielle, sans pour autant rejeter les innovations technologiques qui s'avèrent utiles pour atteindre les normes modernes de sécurité alimentaire. Dans ce contexte, il y a un raisonnement sur la communauté alimentaire qui, bien que selon Slow Food elle ait une référence territoriale explicite, est en réalité un réseau qui comprend une pluralité de sujets qui ne sont pas nécessairement locaux, mais qui travaillent pour la reproduction co-évolutive de la place spécifique de la communauté, à travers la conservation et la valorisation de la nourriture.

5. Critique de la mondialisation. Au fil des ans, Slow Food est devenu le porteur de la préservation des techniques et recettes traditionnelles, en redonnant de la valeur aux chaînes d'approvisionnement locales et aux produits indigènes. Il ne s'agit pas d'opposer innovation et modernité, mais de s'en détacher lorsqu'elle tend à faire prévaloir l'homogénéisation sur la diversité, tant culturelle que biologique, ou lorsque cette diversité devient la propriété de quelquesuns. Slow Food ne veut pas s'opposer au système de la mondialisation mais propose une "mondialisation par le bas ", c'est-à-dire une mondialisation qui implique la structuration d'un développement du secteur agroalimentaire qui rejette l'agro-industrie, respecte la nature, son époque et valorise sa diversité.

C'est seulement grâce à une vision holistique que Slow Food construit sa stratégie en l'appliquant aux différents projets. Slow Food développe une " sociologie de l'alimentation » dans laquelle l'éco-gastronomie, la consommation responsable et la sauvegarde de la diversité sont interconnectées, générant une vision globale (A. Menerei). ${ }^{24}$

\section{Les projets de Slow Food : 1'Arche du Goût}

L'agro-biodiversité souffre et a subi de graves pertes, mais pour protéger ce patrimoine, il est nécessaire de le connaître avant tout. 
À cette fin, le projet Slow Food - l'Arche du Goût - a été lancé en 1996 dans le but de créer une plateforme virtuelle où les produits alimentaires, les variétés végétales et les races animales menacées d'extinction pourraient être connus. Aujourd'hui, plus de 5000 produits sont recensés dans plus de 121 pays, incluant des espèces animales et végétales, mais aussi des processus de production réunis dans un seul projet qui témoigne du savoir du monde agricole et artisanal transmis au fil du temps. L'objectif est de promouvoir la participation de l'interlocuteur et de l'inviter à interagir de manière active : connaître le produit, le consommer, soutenir les producteurs, favorisant ainsi sa conservation et sa reproduction. L'objectif n'est pas de cataloguer le matériel génétique, mais plutôt de créer une interaction avec celui-ci, afin de redécouvrir et de réévaluer les ressources d'un territoire, de manière à créer un réseau local et en même temps international non seulement comme soutien économique, mais, plus important encore, comme soutien social. ${ }^{25}$

L'Arche du Goût veut être un point de référence à partir duquel il est possible de développer à la fois des projets liés au réseau Slow Food et des initiatives externes parallèles. Par ce biais, la capacité de diffuser des connaissances liées à une communauté, capable de déterminer sa propre survie, est ainsi reconnue. Conformément à la mission de Slow Food, le projet de l'Arche du Goût pose les bases de la création d'un réseau. La réflexion sur laquelle se développe ce désir est la nécessité de trouver des informations non seulement par les canaux conventionnels, comme l'analyse des travaux de recherche qui se réferent au domaine d'intérêt, la consultation de catalogues de variétés et de races, de livres de recettes qui contiennent des informations utiles, mais aussi la mise en réseau avec les jardins botaniques, les banques de germoplasme, les collections variétales des écoles d'agriculture, des universités et des centres de recherche. Et, de fait, en s'appuyant directement sur le contact avec le territoire, car les informations nécessaires sont souvent liées aux connaissances traditionnelles. Des organismes de référence et des experts dans le domaine apportent leur contribution, soutenus par un contact direct avec la communauté d'origine. Les collaborateurs de la fondation s'occupent d'eux, passent au crible le matériel fourni et encouragent eux-mêmes la recherche. L'interlocuteur avec lequel Slow Food travaille en interface est défini comme un « signaleur ", qui est reconnu pour sa proactivité en plaçant son nom à côté du produit signalé sur le site web de l'association. La difficulté initiale de ce projet aura été de créer des paramètres universellement reconnaissables, des contenus accessibles à tous, simples mais complets, afin de trouver les informations nécessaires pour évaluer le statut d'un produit, dans lesquels chacun peut s'orienter pour pouvoir impliquer des individus dans l'initiative. ${ }^{26}$

25 Slow Food Foundation, Ark of Taste, What we do?, 2018.

26 https://www.fondazioneslowfood.com/it/cosa-facciamo/arca-del-gusto/il-progetto/storia/. 
Afin d'identifier un produit à déclarer, il est nécessaire de fournir des informations détaillées afin que les exigences pertinentes puissent être définies. La particularité d'un produit peut être reconnue dans un petit détail : une espèce rare, un type de fermentation ou de cuisson inhabituel, ou la méthode de production, mais aussi la valeur sociale d'un produit transmis dans le temps. Dès que le mécanisme qui intéresse un produit particulier est déclenché, il doit faire l'objet d'une enquête approfondie et être complété. Les paramètres recherchés subissent de légères variations spécifiques inhérentes au type de produit en question, remettant en cause la nature du produit, qu'il soit le produit d'une domestication, d'une espèce sauvage ou d'un produit transformé. Les productions sont le résultat d'un savoir artisanal transmis au fil du temps dans toutes les régions du monde. Ils reflètent la culture d'un lieu, les besoins et les outils. Les petites variations de production peuvent générer une différence reconnaissable dans le produit fini ; tout comme l'utilisation d'une matière première donnée dans une production est une composante tangible du territoire et de la culture dans lesquels elle est insérée. Il est donc nécessaire d'identifier les caractéristiques qui rendent le produit unique. Unique en son genre, l'abricot Rouget de Sernach se caractérise par un arôme parfumé et un goût particulièrement doux, dû à un mélange d'acides et de sucres inhabituel pour ce fruit. L'abricot Rouget de Sernach a une peau rouge et une chair succulente. En outre, pour comprendre et définir le lien entre un produit et la région dans laquelle il est cultivé, il ne suffit pas de le définir comme « local ». ${ }^{27}$

Les producteurs locaux peuvent introduire et utiliser des variétés exogènes, des hybrides ou des produits non liés à la culture locale. Le territoire ne se contente pas de donner au produit ses caractéristiques chimiques et physiques, mais le situe également dans un contexte culturel et historique. Par exemple, il est reconnu que la zone de production de l'abricot Rouget coïncide avec la zone A.O.C. Pays d'Uzèges-Pont du Gard. Ce cultivar local a été découvert par hasard dans les années 1930 par un agriculteur local, Laurent Beylesse, surnommé Rouget.

Enfin, deux autres paramètres fondamentaux sont analysés et évalués afin qu'un produit puisse faire partie du projet Arche du Goût : la qualité et la quantité. La qualité agroalimentaire est un concept complexe qui a évolué au fil du temps; elle peut se référer à différentes caractéristiques du produit, c'est pourquoi il n'est pas possible de donner une définition univoque. Alors que la quantité produite est un indice de la diffusion sur le territoire, comme reflet du risque potentiel d'extinction auquel un produit peut être exposé. Si l'on prend l'exemple ci-dessus, la commercialisation de l'abricot Rouget a commencé au début des années 1940, mais la plupart des vergers ont toujours été destinés à un usage familial. En 2005, seuls 14 producteurs cultivaient encore l'abricot Rouget, alors qu'aujourd'hui la production est en hausse, capable de garantir 
plus de 15 hectares de vergers, et varie de 12 à 20 tonnes chaque année. Il est difficile de conserver la fraîcheur du produit, c'est pourquoi la plupart des fruits sont utilisés pour produire des jus et des confitures.

Comme indiqué précédemment, l'intérêt est centré sur le produit, il n'est donc pas intéressant pour le projet d'identifier précisément les chiffres et les quantités de référence, il faut un ordre de grandeur dans lequel placer la production, pour vérifier la nature elle-même. ${ }^{28}$

Un exemple intéressant qui permet de développer une réflexion sur ce sujet est l'histoire et la production de l'angélique confite de Limagne. ${ }^{29}$

L'Angelica archangelica est une plante de la famille des Apiacées, également appelée herbe des anges, probablement originaire de l'Himalaya. C'est une plante bisannuelle, dont les graines semblent avoir été apportées en Europe par les Croisés au XI siècle. Elle s'est bien adaptée à la riche plaine de Limagne. Très aromatique, elle a parfumé l'actuel quartier de la Plaine à Montferrand. Le pétiole (la partie à la base de la feuille, avant le pédoncule) et le pédoncule sont traditionnellement utilisés en confiserie pour fabriquer des fruits confits et des pâtisseries aux fruits.

Au début du $\mathrm{XX}^{\mathrm{e}}$ siècle, les terres de Limagne ont été le premier centre de production d'angélique confite, destinée notamment à l'exportation vers les pays anglo-saxons. Les principaux acheteurs d'angélique locale sont, à l'époque, les confiseurs Aubert, Gauffridy, Cruzilles, Dischamp et Humbert. C'est ainsi que les confiseries de la région de Clermont-Ferrand, grâce notamment à l'angélique, ont acquis une renommée internationale. Aujourd'hui encore, chaque année, le premier samedi de décembre, la fête de l'angélique a lieu à Montferrand. Si dans les années 1950, la Limagne produisait 250 tonnes d'angélique, aujourd'hui, on ne récolte plus que 2 tonnes de pétioles par an sur un champ d'environ 0,5 hectare à La Sauvetat, vendus exclusivement à la société Cruzilles, derniers producteurs de fruits confits en Auvergne. Traditionnellement en confiserie, l'angélique était utilisée pour décorer les gâteaux, mais aujourd'hui elle a été remplacée par l'hostie, qui coûte moins cher, tandis que les fruits confits et les pâtisseries aux fruits sont de moins en moins consommés. C'est une cuisinière française, Eva Muller, qui a proposé de diffuser l'histoire de ce produit. Il est certain qu'une caractéristique importante qui rend le projet de l'Arche du Goût unique en soi est la collaboration active de la société civile, puisque tout citoyen peut se joindre à l'initiative en jouant le rôle de "signaleur ". Ainsi, le projet est réalisé grâce à la nécessaire proactivité des communautés qui, composées d'individus, représentent chacune leur propre territoire d'origine. Ces valeurs ont été reprises dans la

28 https://www.fondazioneslowfood.com/it/cosa-facciamo/arca-del-gusto/criteri-per-lasegnalazione/.

29 https://www.fondazioneslowfood.com/it/arca-del-gusto-slow-food/angelica-candita-dilimagne/. 
dernière initiative à laquelle Slow Food a participé, toujours liée à ce projet. À l'occasion de l'Année européenne du patrimoine culturel 2018, l'Union européenne a promu et financé le développement de diverses initiatives, dans le but d'encourager les citoyens à s'engager en faveur du patrimoine culturel européen, en renforçant leur sentiment d'appartenance à un espace commun : découvrir sa culture passée pour promouvoir l'avenir de l'union. Grâce à cette initiative, le projet " Food is Culture » est né, entièrement dédié au projet Arche du Goût de Slow Food. Les objectifs sont de faire comprendre aux citoyens européens que le patrimoine alimentaire est un moyen d'exprimer leur appartenance à l'Europe et de mieux comprendre la richesse et la spécificité de sa diversité culturelle. Communiquer l'importance culturelle de la gastronomie par l'innovation et l'interaction entre les secteurs culturels et créatifs. Et enfin pour sauvegarder et valoriser le patrimoine alimentaire européen. ${ }^{30}$

\section{Projets Slow Food : les Sentinelles}

Comme démontré jusqu'à présent, l'Arche du Goût combine l'importance des aspects agronomiques avec les aspects socioculturels d'un produit. Le projet Sentinelles, d'autre part, est le résultat d'une nouvelle mise en œuvre opérationnelle de l'Arche du Goût, qui se caractérise par une structure plus complexe, qui détermine des impacts environnementaux, sociaux, économiques et culturels plus importants.

Le projet Sentinelles a été lancé en 2000 pour sauvegarder et relancer au niveau international les petits produits artisanaux menacés d'extinction. En 2003, grâce à la création de la Fondation Slow Food pour la Biodiversité, le projet est devenu, avec l'Arche du Goût, l'une des principales activités, touchant aujourd'hui 575 Sentinelles dans plus de 70 pays du monde. ${ }^{31}$

Par ses activités de protection, de sauvegarde et de promotion, le projet Sentinelles a activement contribué au sauvetage et à la récupération de nombreuses variétés végétales, de races animales indigènes et de produits transformés. Grâce au réseau, les traditions et les connaissances locales ont été maintenues, générant un échange mutuel de connaissances. Le soutien à la production a également favorisé la croissance des économies locales, en stimulant le marché, en garantissant aux communautés une plus grande sécurité alimentaire, la souveraineté alimentaire et le bien-être général.

Pour faire preuve de cohérence et d'alignement avec la philosophie du mouvement, les producteurs sont tenus d'adhérer pleinement à la philosophie du bien, de l'équité et de la propreté en promouvant la durabilité environnementale, socioculturelle et économique.

30 https://www.fondazioneslowfood.com/it/food-is-culture/.

31 Slow food, "Faq: the communities and the new model", 2019 [Online]. https://www.slowfood. com/our-network/slow-food-communities/faq/. 
Pour qu'une sentinelle soit établie, cinq critères généraux mais nécessaires doivent être remplis.

La Sentinelle (produit transformé/variété/race/paysage rural ou écosystème) doit être en danger d'extinction, réel ou potentiel (1). L'évaluation se fait par une analyse quantitative de l'objet en question au moyen d'une comparaison temporelle dans une zone définie. La sentinelle doit assumer un lien étroit avec le territoire (2), c'est-à-dire s'identifier à une zone géographique spécifique d'origine/de culture/d'élevage/de production. Une démonstration claire de cette relation est le cas de la Sentinelle de l'épeautre de Haute-Provence. ${ }^{32}$ Population botanique locale, les premières traces remontent à 9000 ans avant J.-C. et on estime qu'elle provient de la ceinture occidentale de la Turquie actuelle. Depuis des milliers d'années, l'histoire du petit épeautre (Triticum monococcum) est étroitement liée à celle de la civilisation méditerranéenne, qui le consommait en abondance. Cependant, il a été presque totalement remplacé par le blé, qui a pris la place de diverses céréales mineures parce qu'elles n'étaient plus rentables et nécessitaient plus de travail. Ce n'est que ces dernières années que le petit épeautre a recommencé à susciter l'intérêt grâce à sa rusticité, adaptée aux climats semi-arides et aux sols pauvres, et à ses qualités nutritionnelles et organoleptiques. Grâce à l'activisme de plusieurs producteurs qui ont rejoint un consortium, la variété a obtenu la reconnaissance de l'I.G.P. (Indication Géographique Protégée) en 2010. Actuellement, une trentaine de producteurs cultivent cette céréale dans le but de défendre, promouvoir et valoriser une variété ancienne, entrecoupée de champs de lavande et de légumineuses, unique par sa vocation environnementale.

La Sentinelle doit maintenir le lien avec la mémoire et l'identité d'un groupe (3), c'est-à-dire le lien avec la sphère historique, socio-économique et culturelle d'un groupe. Par la domestication d'une race ou d'une variété, au fil des ans, celle-ci s'enracine dans la culture d'un territoire et devient ainsi partie intégrante de l'identité territoriale et de la mémoire collective. Un exemple peut être la production de fromages dans les alpages, comme dans le cas du fromage Malga del Béarn. ${ }^{33}$ La tentation de quitter les alpages et de se limiter à travailler dans la vallée existe, avec le danger, cependant, de perdre ces paysages et les connaissances anciennes liées à la production de fromage. Les fromages de Malga del Béarn sont un signe évident de cette résistance. Grâce à la création de la Sentinelle, qui réunit environ 80 bergers transhumants de trois vallées, l'objectif est de lancer un programme de promotion des produits des alpages à la recherche d'une qualité excellente, en préservant l'authenticité du goût et en obtenant une plus grande reconnaissance. La Sentinelle doit être une production de quantités limitées (4), respectant les pratiques et techniques traditionnelles des petites entreprises artisanales. Enfin, elle doit répondre à la

32 https://www.fondazioneslowfood.com/it/presidi-slow-food/piccolo-farro-dellalta-provenza/.

33 https://www.fondazioneslowfood.com/it/presidi-slow-food/formaggi-di-malga-del-bearn/. 
qualité organoleptique, mais aussi environnementale et sociale (5), toujours en référence à la qualité, la propreté et l'équité, et donc à la durabilité environnementale, économique et socio-culturelle. ${ }^{34}$

Pour qu'une Sentinelle puisse démarrer et répondre aux cinq critères, il est nécessaire de remplir un formulaire de candidature préparé par l'association (informations de base sur la chaîne de production et les producteurs), de le partager avec la communauté et de l'envoyer à la Fondation pour la biodiversité Onlus (ONLUS = Organizzazione Non Lucrativa di Utilità Sociale, organisation à but non lucratif). Si le rapport est valable, une visite et une rencontre avec les producteurs et les correspondants locaux concernés seront organisées. Une fois que l'on a brossé un tableau organique de la situation, on peut commencer à élaborer les règlements de production et à choisir le nom de la Sentinelle en tenant compte de l'identité historique et de la zone de production. Jusqu’à présent, les pays qui ont réglementé le label «Sentinelle Slow Food $^{\circledR}$ "(Italie, y compris le café et le cacao traités en Italie mais provenant du Sud du monde, la Suisse) ont bénéficié du label pour les Sentinelles qui ont régulièrement établi les règlements de production. Cependant, Slow Food se trouve aujourd'hui dans une phase de transition en ce qui concerne le logo et son utilisation, nous nous limiterons donc à ce qui a été dit jusqu'à présent. Une Sentinelle est la " traduction » cohérente de la philosophie de Slow Food, il doit faire preuve de transparence par le biais de l'assurance qualité. Cette garantie a, jusqu’à présent, été « autocertifiée » par des producteurs individuels utilisant différents outils.

Les lignes directrices, élaborées par la Fondation Slow Food pour la Biodiversité, en collaboration avec les techniciens et les producteurs, sont des lignes directrices pour chaque catégorie de produits (produits de boulangerie, races autochtones à viande, conserves de fruits et légumes, fromages et races laitières, sel, etc. Ces dernières sont le document exhaustif établi et signé par le groupe de producteurs : il est systématiquement structuré en articles qui expliquent clairement et complètement les aspects de la production. Il contient également un article sur les " contrôles " précisant, si la Sentinelle est soumise à une certification supplémentaire, les contrôles pertinents auxquels il pourrait être soumis. Bien que Slow Food ait une confiance totale dans les producteurs et les structures locales, elle peut en tout cas effectuer des visites inopinées dans la zone et mener d'éventuelles activités de contrôle. Comme mentionné précédemment, la qualité des Sentinelles est actuellement garantie par l'auto-certification des producteurs ; cependant, l'association est actuellement engagée dans un projet pilote qui lui permet d'évaluer l'adaptabilité d'un outil de certification de la qualité plus " personnalisé ", construit ad hoc sur la base de la structure de l'organisation : le Système de Garantie Participatif.

34 Slow food, "Faq: the communities and the new model", 2019, https://www.slowfood.com/ our-network/slow-food-communities/faq/. 
En outre, une étiquette narrative est prévue pour les Sentinelles. Selon Slow Food, la qualité d'un produit alimentaire est avant tout un récit ; c'est pourquoi elle a décidé de fournir, outre les indications requises par la loi, un modèle d'étiquetage qui peut fournir des informations sur le territoire, la technique de culture, la transformation, les méthodes de conservation et, bien sûr, les caractéristiques organoleptiques et nutritionnelles ; il n'est pas obligatoire mais constitue un outil de communication innovant que le producteur pourrait utiliser pour la commercialisation.

Enfin, depuis 2008, le label "Sentinelle Slow Food" " a été mis en place, ce qui assure au consommateur que le produit Sentinelle doit être considéré comme tel. C'est pourquoi un cahier des charges de production a été établi, basé sur le respect de la tradition et la durabilité environnementale, socioéconomique et culturelle.

Comme il a été souligné jusqu'à présent, l'approche, les objectifs de l'établissement des Sentinelles entre les différents continents, en Europe, en Amérique ou en Afrique, sont les mêmes ; cependant, il est inévitable de dire que dans des contextes sociaux, économiques, culturels et politiques aussi différents, les projets prennent des formes différentes, dans lesquelles les relations réciproques entre les composantes environnementales, techniques, sociales et économiques changent afin de tirer le meilleur parti du potentiel exprimé par la communauté et le territoire concerné. On peut donc dire que le projet des Sentinelles représente un modèle, car son application devrait déclencher un exemple de production vertueuse qui peut être appliqué à d'autres produits et diffusé à d'autres générations, afin de garantir la subsistance alimentaire parallèlement à la commercialisation de la production excédentaire.

Dans ce mécanisme, le rôle central que Slow Food maintient en tant que pivot et point de référence pour le réseau pourrait être identifié comme une faiblesse possible, créant un lien et une dépendance excessifs des communautés alimentaires par rapport à l'organisation elle-même ${ }^{35}$.

\section{Projets visant à sauvegarder et à promouvoir la biodiversité dans un cadre intégré de durabilité environnementale, économique, sociale et culturelle}

Les grandes lignes des projets de l'Arche du Goût et des Sentinelles partent de la philosophie générale de Slow Food, qui se reflète évidemment dans toutes ses actions.

35 Egidio Dansero, Cristiana Peano, Carlo Semita, Nadia Tecco, "Il modello delle comunità del cibo nell'azione di Slow Food in Africa. Modalità operative e indicazioni per la valutazione e il monitoraggio delle attività", 2015, https://www.slowfood.com/sloweurope/wp-content/ uploads/rapporto_11maggio.pdf. 
L'organisation ne se préoccupe pas tant de diffuser un modèle de conception " conservateur " en ce qui concerne les techniques de production et les connaissances locales, mais plutôt de soutenir un modèle de réinterprétation, de redistribution et de réappropriation des valeurs d'usage, intrinsèques aux ressources naturelles et humaines présentes sur le territoire, à partir de l'interaction de ces dernières avec le contexte local et global, de dialogue entre la spécificité endogène et les stimuli de l'extérieur.

Bien qu'il implique une pluralité de facteurs (environnementaux, sociaux, économiques, culturels, politiques, géographiques), le concept de durabilité adopté et promu par Slow Food peut être essentiellement articulé dans les trois dimensions fondamentales qui composent la durabilité dans sa complexité : environnementale, économique et sociale.

La durabilité environnementale signifie la capacité à maintenir la qualité et la reproductibilité des ressources naturelles dans le temps, à préserver la biodiversité et à garantir l'intégrité des écosystèmes. ${ }^{36}$

L'aspect environnemental est certainement le point de départ des projets nés pour sauvegarder la biodiversité et stimuler la production agroalimentaire durable. L'approche est liée aux principes de la vocation environnementale, des connaissances locales, de l'application de techniques (traditionnelles et modernes) adaptées aux différentes conditions agro-pédoclimatiques liées à la bonne gestion des ressources naturelles (biodiversité, sol, eau). Là où des voies antérieures ou des connaissances locales ont permis le développement de l'agriculture biologique (entendue non pas exclusivement en termes de certification mais comme des techniques agronomiques), le développement d'une Sentinelle se concentre sur le renforcement des concepts de contrôle des cultures biologiques et la diffusion de cette philosophie. À l'inverse, là où l'agriculture conventionnelle joue encore un rôle important dans la gestion des cultures, le projet Sentinelles vise à accompagner les producteurs sur la voie d'une plus grande durabilité environnementale, en convertissant progressivement les modèles agricoles conventionnels en modèles durables. Enfin, conformément à la recherche d'un environnement durable, les thèmes du bien-être animal, des économies d'énergie et de l'emballage sont inclus. ${ }^{37}$

En ce qui concerne la durabilité économique, cela signifie la capacité à générer des revenus et à travailler de manière durable et à atteindre l'écoefficacité, c'est-à-dire une utilisation rationnelle des ressources disponibles, comme la réduction de l'exploitation des ressources non renouvelables. ${ }^{38}$

36 Ibid.

37 C. Peano, N. Tecco, E. Dansero, V. Girgenti, F. Sottile, Evaluating the Sustainability in Complex Agri-Food Systems: The SAEMETH Framework. Sustainability 7(6), 2015, 6721-6741, https:// doi.org/10.3390/su7066721.

38 Egidio Dansero, Cristiana Peano, Carlo Semita, Nadia Tecco, "Il modello delle comunità del cibo nell'azione di Slow Food in Africa. Modalità operative e indicazioni per la valutazione 
Une partie de l'effort de l'association en matière de durabilité économique consiste à accroître la visibilité des petits producteurs.

Les objectifs finaux sont en effet liés à une meilleure rémunération des producteurs, à la possibilité d'augmenter les ventes, mais aussi à une augmentation de l'emploi direct ou dans des secteurs complémentaires comme le tourisme.

Les aspects économiques du projet Sentinelles sur les territoires ont fait l'objet de recherches par l'Université Bocconi de Milan (Antonioli Corigliano et Viganò, 2002). L'étude, réalisée sur 54 Sentinelles italiennes de différents types, a montré un impact économique considérable qui implique à la fois un changement en termes de quantité et de qualité de la production et en termes de prix de vente. Une expérience similaire a été répétée en 2006 (Baggi, 2007) avec un nouveau questionnaire proposé à 31 Sentinelles (18 Sentinelles italiennes, 6 du reste de l'Europe, 3 d'Amérique centrale et du Sud, 2 d'Asie et d'Afrique).

Les résultats ont suscité un intérêt particulier si l'on considère que dans le cas des Sentinelles italiennes, dans certains cas comme celui des légumineuses, les prix de vente sont plus que doublés. L'objectif d'une Sentinelle, d'un point de vue économique, est donc de parvenir à un accord de prix équitable entre le producteur et le consommateur. ${ }^{39}$

Le résultat de cet accord est la Sentinelle du Noir de Bigorre, la plus ancienne race de porc connue en France. Alors qu'il y avait encore 28000 porcs reproducteurs de la race Noir de Bigorre dans les années 1930, il n’en reste que quelques centaines 40 ans plus tard. La race était perdue en raison de sa faible adaptabilité à la reproduction intensive et aux croisements fréquents. Grâce au recensement de 1981 de 34 truies de race pure, les producteurs et les techniciens ont pu empêcher leur extinction en premier lieu, mais le défi était de trouver de nouveaux débouchés commerciaux pour justifier leur élevage.

Ce n'est qu'en 1992 que la chaîne des Hautes-Pyrénées s'est enfin réunie - de l'élevage à la charcuterie - et que la production de salaisons de qualité a commencé. Aujourd'hui, le produit transformé le plus important obtenu à partir du porc est le jambon, mais il existe également d'autres spécialités de charcuterie et de viande fraîche qui permettent à un petit groupe de producteurs de faire de cette production une activité commerciale. ${ }^{40}$

La durabilité sociale, quant à elle, signifie la capacité à garantir l'accès à des biens considérés comme fondamentaux (sécurité, santé, éducation) et à des conditions de bien-être (plaisir, sérénité, socialité), de manière équitable

e il monitoraggio delle attività, 2015, https://www.slowfood.com/sloweurope/wp-content/ uploads/rapporto_11 maggio.pdf.

39 M. Antonioli Corigliano and G. Viganò, "I Presidi Slow Food: da iniziativa culturale ad attività imprenditoriale", Il Sole24Ore, 2002.

40 https://www.fondazioneslowfood.com/it/presidi-slow-food/maiale-nero-di-bigorre/. 
au sein de la communauté. Si, par exemple, la durabilité économique d'une Sentinelle est plus définissable en termes universels - cela ne signifie pas qu'elle le soit d'un point de vue pratique - il est plus complexe, compte tenu des différences socioculturelles qui caractérisent chaque zone géographique, de définir la durabilité sociale. Les actions entreprises ces dernières années dans les pays du Sud ont souligné l'importance nécessaire à réserver à ces aspects. Il est clair qu'une amélioration des revenus des producteurs peut se traduire par une meilleure qualité de vie et un meilleur accès aux services (soins médicaux, éducation, hygiène). Toutefois, pour les Sentinelles d'un pays du Nord du Monde, des aspects tels que l'accès aux services sont moins évidents car la plupart d'entre eux sont déjà présents.

Enfin, la durabilité sociale s'accompagne d'aspects culturels, fortement liés à la capacité des personnes actives dans les projets à retracer la culture locale. En impliquant également de nouveaux sujets du territoire, tels que les étudiants, les restaurateurs, les autorités locales, les associations, l'objectif est de se " rétablir " dans ses propres origines et dans son histoire; par une communication efficace, il est ainsi possible, à partir par exemple d'un produit agroalimentaire, de transmettre un sentiment d'appartenance à une communauté, définissant ainsi une identité culturelle. Bien sûr, cela peut se traduire par la récupération d'événements historiques, des interventions architecturales et plus généralement par la promotion du tourisme durable; tout cela générera des effets positifs sur le territoire et sur la communauté elle-même. ${ }^{41}$

Afin de donner une plus grande crédibilité aux projets et à l'action de Slow Food, l'association a également développé une grille d'évaluation de la durabilité dont l'objectif est d'entreprendre une action de suivi dans le temps qui permette des évaluations systématiques et transversales de l'avancement des projets.

En partant des trois dimensions et sous-catégories identifiées ci-dessus, c'est-à-dire les dimensions et sous-catégories socioculturelles, environnementales et économiques, la mesure de la valeur associée est quantitative dans certains cas et qualitative dans d'autres. Les indicateurs identifiés sont : a) des indicateurs capables d'exprimer la distance entre la situation réelle et celle considérée comme durable selon la compréhension de Slow Food ; b) des indicateurs ayant le bon degré de sensibilité pour signaler correctement les changements du phénomène qu'ils décrivent ; c) des indicateurs efficaces, adaptés au contexte et à l'articulation des activités de suivi.

Il convient de noter que la source d'information privilégiée pour la compilation du formulaire de suivi est l'entretien ou l'interview orale, qui doit être conduit auprès d'un certain nombre d'interlocuteurs privilégiés en fonction de la taille et du nombre de participants au projet.

41 Cristiana Peano, "I progetti della fondazione Slow Food per la biodiversità: un modello per valorizzare cibi locali di qualità", in Geografia da e para a cooperação ao desenvolvimento territorial: experiencias brasileiras e italianas, Ed. Outras Expressóes, 2011, p. 293-313. 
En tenant compte des différentes dimensions de la durabilité (aspects économiques, sociaux, environnementaux et culturels) et de leur déclinaison dans le contexte spécifique du réseau Slow Food, la grille d'indicateurs développée à partir d'expériences concrètes d'application devrait permettre de vérifier la faisabilité du projet, de suivre l'avancement des actions prévues, à réaliser dans le futur et de déclencher une activité vertueuse de production de données, ainsi qu'un modèle de rapport de durabilité. ${ }^{42}$

Dans tous les projets Slow Food, le résultat de l'interaction entre la nature et la culture est largement mis en avant, dans lequel la société civile est reconnue comme jouant un rôle décisif dans la protection et la promotion du patrimoine agroalimentaire. Dans ce paysage culturel, les communautés agricoles, par leurs activités quotidiennes, sont l'expression de la différence et de l'interaction constante entre les processus naturels et culturels. On peut donc dire que les produits alimentaires sont le résultat de connaissances et de technologies complexes, ce qui définit la culture comme un point d'intersection entre la tradition et l'innovation. C'est la tradition car elle est constituée de connaissances, de techniques et de valeurs qui sont transmises ; c'est l'innovation car ces connaissances, techniques et valeurs modifient le rôle de l'homme dans les processus naturels, le rendant capable de s'adapter à de nouvelles formes. ${ }^{43}$

Le dynamisme des processus culturels est également caractéristique des processus écosystémiques, dont la relation permet à l'agro-biodiversité de subsister et de se préserver. Bien que ce dynamisme puisse sembler un concept marginal au sein du projet Arche du Goût, il en est le cœur battant. Ce projet permet l'analyse et le développement de telles dynamiques dans le but de permettre leur réalisation, par le biais de la diffusion.

Dans le panorama actuel, communiquer sur l'importance culturelle de l'alimentation par des approches intersectorielles et multisectorielles permet de générer une diversité qui devient encore plus incisive et transversale.

L'introduction des marchés financiers et l'homologation des modèles alimentaires ont conduit à une nouvelle focalisation sur les cultures locales. Bien qu'elle ait toujours existé, la territorialité en tant que concept et connotation positive est une interprétation récente. Ce phénomène a permis ce que l'on appelle une "redécouverte des racines ", promue par les projets Slow Food présentés jusqu’à présent. En fait, les projets Sentinelles et Arche du Goût visent à redonner de l'importance aux produits et aux producteurs pour la valeur qu'ils sont eux-mêmes capables de générer.

42 C. Peano, N. Tecco, E. Dansero, V. Girgenti, F. Sottile, Evaluating the Sustainability in Complex Agri-Food Systems: The SAEMETH Framework. Sustainability 7(6), 2015, 6721-6741, https:// doi.org/10.3390/su7066721.

43 M. Montanari, Il cibo come cultura. Economica Laterza, Roma, Laterza Editore, 2004. 
Dans toutes les sociétés traditionnelles, l'alimentation devient le premier facteur de différenciation sociale. Lorsque, finalement, la nourriture devient une marchandise accessible à tous, ce modèle perd de sa valeur et le territoire est remplacé par une expression de différenciation. ${ }^{44}$

En ce qui concerne le projet Sentinelles, il a une forte connotation locale car il insiste sur des territoires spécifiques où des systèmes agroalimentaires de valeur ont été identifiés. En dépit des différences parfois mises en évidence dans les différents itinéraires, il ne fait aucun doute que tous les projets sont l'activation d'un processus territorial dynamique qui contribue non seulement à la promotion des produits locaux traditionnels mais aussi à la reconstruction des itinéraires socioculturels.

Ils fournissent une stratégie pour les producteurs qui souhaitent rester ou retourner sur des territoires complexes d'un point de vue productif mais aussi socioculturel. ${ }^{45}$

La contemporanéité, dominée par l'instabilité et les crises sociales mais aussi environnementales, nous impose de poursuivre des modèles d'innovation et de développement. Le " retour aux origines " dont il est question n'est pas un simple retour en arrière de la société humaine vers le passé, mais c'est une réappropriation de valeurs perdues, qui prennent nécessairement de nouvelles formes et configurations, adaptées au panorama contemporain, en mouvement continu.

Cristiana Peano

Dipartimento di Scienze Agrarie, Forestali e Alimentari (DISAFA) Università degli Studi di Torino, Association Slow Food International

Anna Gregis

Dipartimento di Culture, politiche e società (CPS) Università degli Studi di Torino Cattedra UNESCO in Sviluppo Sostenibile e Gestione del Territorio

Chiara Ghisalberti Dipartimento di Scienze Agrarie, Forestali e Alimentari (DISAFA) Università degli Studi di Torino - Italie

44 Massimo Montanari, La fame e l'abbondanza. Storia dell'alimentazione in Europa, Ed. 12, Laterza, 2019.

45 Cristiana Peano, "I progetti della fondazione Slow Food per la biodiversità: un modello per valorizzare cibi locali di qualità", in Geografia da e para a cooperação ao desenvolvimento territorial: experiencias brasileiras e italianas, 2011, p. 293-313, Ed. Outras Expressóes. 


\section{Résumé}

Le rôle fondamental de la préservation et de la sauvegarde de l'agro-biodiversité est caché dans la relation entre la nature et la culture. L'article vise à présenter deux cas spécifiques, les projets de l'Arche du Goût et des Sentinelles, promus par l'association Slow Food International dont l'ambition est de préserver les races autochtones et les variétés végétales locales, de soutenir les produits de qualité, les territoires, les écosystèmes fragiles et/ou complexes, en récupérant les méthodes de transformation traditionnelles. Grâce à une action intersectorielle et à une analyse intégrée des écosystèmes, y compris les composantes naturelles et anthropiques, il sera donc possible de renforcer la diversité biologique.

\section{Mots-clés}

Agro-biodiversité, systèmes agricoles diversifiés, durabilité, Fondation Slow Food, communauté alimentaire, approche intégrée intersectorielle, identité alimentaire locale.

\section{Abstract}

The fundamental role of preserving and safeguarding agro-biodiversity is concealed in the relationship between nature and culture. The article aims to present two specific cases, the Ark of Taste and Presidia projects, promoted by the Slow Food internation association which ambition is to preserve native breeds and local plant varieties, support quality products, fragile andlor complex territories and ecosystems, recovering traditional processing methods. Through intersectoral action and an integrated analysis of ecosystems, including both natural and anthropic components, it will therefore be possible to enhance biological diversity.

\section{Keywords}

Agro-biodiversity, Diversified Farming Systems, Sustainability, Slow Food Foundation, Food community, Integrated (intersectoral) Approach, Local food identity. 